\title{
Pathogenic Bacillus anthracis in the progressive gene losses and gains in adaptive evolution GX Yu
}

Address: Dept. of Biological Science and Dept. of Computer Science, Boise State University, Boise, Idaho 83725, USA

Email: GX Yu - gongxinyu@boisestate.edu

from The Seventh Asia Pacific Bioinformatics Conference (APBC 2009)

Beijing, China. 13-16 January 2009

Published: 30 January 2009

BMC Bioinformatics 2009, I0(SuppI I):S3 doi:I0.1 186/I47I-2105-I0-SI-S3

This article is available from: http://www.biomedcentral.com/I47I-2/05/I0/SI/S3

(C) 2009 Yu; licensee BioMed Central Ltd.

This is an open access article distributed under the terms of the Creative Commons Attribution License (http://creativecommons.org/licenses/by/2.0), which permits unrestricted use, distribution, and reproduction in any medium, provided the original work is properly cited.

\begin{abstract}
Background: Sequence mutations represent a driving force of adaptive evolution in bacterial pathogens. It is especially evident in reductive genome evolution where bacteria underwent lifestyles shifting from a free-living to a strictly intracellular or host-depending life. It resulted in lossof-function mutations and/or the acquisition of virulence gene clusters. Bacillus anthracis shares a common soil bacterial ancestor with its closely related bacillus species but is the only obligate, causative agent of inhalation anthrax within the genus Bacillus. The anthrax-causing Bacillus anthracis experienced the similar lifestyle changes. We thus hypothesized that the bacterial pathogen would follow a compatible evolution path.

Results: In this study, a cluster-based evolution scheme was devised to analyze genes that are gained by or lost from B. anthracis. The study detected gene losses/gains at two separate evolutionary stages. The stage $I$ is when $B$. anthracis and its sister species within the Bacillus cereus group diverged from other species in genus Bacillus. The stage II is when B. anthracis differentiated from its two closest relatives: $B$. cereus and $B$. thuringiensis. Many genes gained at these stages are homologues of known pathogenic factors such those for internalin, $B$. anthracis-specific toxins and large groups of surface proteins and lipoproteins.

Conclusion: The analysis presented here allowed us to portray a progressive evolutionary process during the lifestyle shift of $B$. anthracis, thus providing new insights into how $B$. anthracis had evolved and bore a promise of finding drug and vaccine targets for this strategically important pathogen.
\end{abstract}

\section{Background}

Genome reduction and gene acquisition in adaptive bacterial evolution: Two sides of coins

Sequence mutations represent a driving force of adaptive evolution in bacterial pathogens. They allow the patho- gens to survive and prosper within the host immune systems and to develop unique host specificity [1-4]. It is especially evident in reductive genome evolution where bacteria underwent dramatic lifestyles shifting from a freeliving to a strictly intracellular or host-associated life [5-7]. 
It results in loss or modification-of-function mutations [8]. The results are a loss of many of the genes and the reduction of bacterial genome size $[8,9]$. The acquisition of virulence gene clusters is another effective strategy in the adaptive evolution of bacterial pathogens from nonpathogenic ancestors $[10,11]$.

The anthrax-causing bacillus species experienced the similar lifestyle changes and likely followed compatible evolution paths. B. anthracis shares a common soil bacterial ancestor with $B$. cereus, $B$. thuringiensis and other closely related bacillus species but it is the only obligate, causative agent of inhalation anthrax within the genus Bacillus $[12,13]$. The bacteria exists in the environment as weatherproof, dormant spores [14] that germinate only after being picked up by macrophages. From there, the $B$. anthracis spores are carried through lung tissue to the regional lymph nodes. During this process, the spores survive and germinate into vegetative bacilli $[15,16]$. The vegetative bacteria then multiply and penetrate into the blood circulation by disrupting the macrophages, leading to massive septicemia [17]. The dramatic lifestyle change from a soil bacterium to an obligate pathogen would put $B$. anthracis and its genome under a strong selective pressure. This study addressed questions on what occurred in gene content during the adaptive evolution and how they impacted on the pathogenesis of $B$. anthracis.

Comparative analyses of completely sequenced genomes of the closely related Bacillus organisms have offered a unique opportunity to answer these questions. With such comparison, Read et al, 2003 were able to show that $B$. anthracis had a reduced capacity for sugar utilization and an expanded array of iron-acquisition genes when compared with $B$. subtilis [18]. Otherwise, almost all putative chromosomal virulence and surface proteins of $B$. anthracis were shown to have homologues in $B$. cereus. Rasko et al, 2004 showed that B. cereus ATCC 10987, a non-lethal dairy isolate in the same genetic subgroup as Bacillus anthracis, contained a single large plasmid [19]. Interestingly, it has similar gene content and genome structure to B. anthracis pXO1 but lacks the pathogenicity-associated, anthrax lethal and edema toxin complex genes. Recently, Han et al, 2006 revealed more systematic differences between $B$. anthracis and its closest relatives: $B$. cereus and $B$. thuringiensis [20]. This genome has complete gene sets that are necessary to encode tripartite lethal toxin and polyglutamic acid capsule; moreover, its flagellar genes are the most fragmental and functional PlcRs are absent. The analyses thus provided a basic understanding of molecular mechanisms of evolution and pathogenesis.

In this study, a cluster-based evolution scheme was devised to analyze genes that are gained by or lost from $B$. anthracis. The section of methods and materials has a detailed description about the scheme. Briefly, a reference genome (RG) was chosen and compared via Blast analysis to all other Bacillus genomes, named target genomes (TG). Genes that are present at RG but absent at TG were identified. This led to a gene absence/presence matrix with genes as rows and TGs as columns, which is then subjected to clustering analysis to identify lineage-specific gene losses or gains. In this study, B. anthracis Ames Ancestor 0581 was chosen as the RG to identify genes that were gained by B. anthracis, B. cereus ATCC 10987 and B. licheniformis ATCC 14580 as RG for those that were lost. $B$. cereus ATCC 10987, another species in the Bacillus cereus group, is one of the closest species to $B$. anthracis while $B$. licheniformis ATCC 14580, belonging to Bacillus subtilis group, is more remotely related. The gene losses defined from the two organism, we hypothesized, would represent two different stages critical for the evolution of $B$. anthracis. This paper presented the analysis. Overall, the analysis illustrated a progressive evolution behind $B$. anthracis: genes were lost and gained selectively, which, we hypothesized, could be one of the main evolutionary forces that have driven $B$. anthracis to become an effective anthrax pathogen.

\section{Materials and methods Genome sequences}

Reference sequences of 12 bacillus genomes were downloaded from the National Center for Biotechnology Information (NCBI) in June 2006 [21]. Among these are three for $B$. anthracis (B. anthracis Ames, B. anthracis Ames Ancestor 0581, and B. anthracis str. Sterne), three for $B$. cereus (B. cereus ATCC 10987, B. cereus ATCC 14579, and $B$. cereus $\mathrm{E} 33 \mathrm{~L}$ ), two for B. licheniformis (B. licheniformis ATCC 14580 and B. licheniformis DSM_13 ATCC 14580), and one for each species of $B$. subtilis (B. subtilis subsp. Subtilis str. 168), B. thuringiensis (B. thuringiensis konkukian str. 97-27), B. halodurans (B. halodurans C-125) and B. clausii (B. clausii KSM-K16).

\section{Analysis of genes losses/gains}

The analysis came with a three-step procedure. First, a reference genome (RG) was selected, which were then compared with a set of target genomes (TGs) through BLAST analysis. Under the default E-value of 1e-4, any RG genes with no TG homologues were defined as $\boldsymbol{a} \boldsymbol{b}$ (absence) genes. The distribution of $\boldsymbol{a} \boldsymbol{b}$ genes of all RG-TGs pairs was then summarized as an $\mathbf{m}$-by-n matrix where $\mathbf{m}$ is the number of $\boldsymbol{a} \boldsymbol{b}$ genes (rows) and $\mathbf{n}$ is the number of TGs (columns). Values of the matrix are either " 0 " or " 1 " where " 1 " indicates that genes present in RG but absent in TG for given TG-RG pairs and " 0 " indicates the genes that are present in both TG and RG. Second, clustering analyses were performed on the data matrix using Cluster (version 3.0), an open source clustering software implemented for gene expression data analysis [22]. Fol- 
lowing parameters were chosen in the analysis to give best possible results: Hierarchical, cluster on rows (gene) and columns (genomes); Spearman Rank Correlation; and Single linkage. The last step is to visualize trees from the clustering analysis with Java TreeView [23] to identify lineage-specific gene losses/gains. In this analysis, we chose $B$. anthracis Ames Ancestor 0581 as a RG for the lineagespecific genes gained by $B$. anthracis, $B$. licheniformis ATCC 14580 and B. cereus ATCC 10987 as the RG for the lineage-specific genes losses.

\section{Function classifications}

For a better illustration, species-specific genes lost/gained were further characterized based on COG assignments inherited from NCBI genome annotations.

\section{Calculation of selective gene losses/gains}

To determine selective gene gains/losses during the adaptive evolution, the following formula was used to evaluate the occurrences of gene lost/gained among COG function groups:

$$
\text { LGpnt }_{\mathrm{COGi}}=\lg _{\mathrm{COGi}} / \mathrm{LG}_{\mathrm{COG}} * 100 \text {; }
$$

While LGpnt ${ }_{\mathrm{COGi}}$ is the percentage of COG occurrences in genes lost/gained, $\lg _{\mathrm{COGi}}$ is the number of genes lost/ gained from the $\mathrm{i}^{\text {th }} \mathrm{COG}$ and $\mathrm{LG}_{\mathrm{COG}}$ is the total number of genes lost/gained that have COG assignments in given genomes. At the same time, a similar formula was used to calculate the distribution of the COG occurrences in genes over the entire genomes:

$$
\text { pnt }_{\mathrm{COGj}}=\mathrm{x}_{\mathrm{COG}} / \mathrm{X}_{\mathrm{COG}} * 100 \text {; }
$$

While pnt ${ }_{\mathrm{COGj}}$ is the percentage of COG occurrences, $\mathrm{x}_{\mathrm{COG} j}$ is the number of genes in the $\mathrm{j}^{\text {th }} \mathrm{COG}$ and $\mathrm{X}_{\mathrm{COG}}$ is the total number of genes that have COG assignments. The selective gene losses/gains were determined by comparing the LGpnt $_{\mathrm{COGi}}$ with pnt $\mathrm{COGj}_{\mathrm{C}}$. Genes were considered to be selectively lost or gained if LGpnt ${ }_{\mathrm{COGi}}$ is greater than pnt${ }_{\mathrm{COG} j}$ at least two fold where $\mathrm{i}=\mathrm{j}$.

\section{Verifications of B. anthracis-specific sequence variants}

Additional nine Bacillus genomes were downloaded to investigate whether the conclusions about the B. anthracisspecific gene changes can be extrapolated to Bacillus organisms beyond the initial genome sets. The new genomes include $B$. weihenstephanensis KBAB4, B. cereus subsp. cytotoxis NVH 391-98, B. thuringiensis str. Al Hakam, which are available only recently at the NCBI genome site [21]. Also included are six other B. anthracis genomes from the Comprehensive Microbial Resource (CMR) [24]: B. anthracis A0039, B. anthracis A0402, B. anthracis str. Kruger B, B. anthracis Vollum, B. anthracis A0071 Western North America, and B. anthracis strain
A2012. All genomes downloaded from NCBI are completely sequenced and fully annotated but those from CMR vary in status in terms of sequencing and annotations. For example, the sequence of $B$. anthracis A0039 is listed as unfinished in sequencing with 5634 annotated genes/proteins. In contrast, the sequence of $B$. anthracis strain A2012 is listed as complete in sequencing but with only 330 annotated genes/proteins.

\section{Results and discussion \\ Gene gains at stages}

The study chose the B. anthracis Ames Ancestor 0581 as reference genomes (RGs) and then compared with other 11 bacillus genomes (TG). With an E-value of $1 e-4$, the comparisons of TG-RG resulted in a gene absence/presence matrix. Clustering analysis on the matrix obtained a phylogenetic (species) tree that reflects evolutionary relationships of these Bacillus species [25]. Genomes of $B$. anthracis, B. cereus, and $B$. thuringiensis (the Bacillus cereus group), genomes of $B$. subtilis and $B$. licheniformis (the Bacillus subtilis group), and those of $B$. halodurans, and $B$. clausii were clustered in three separate phylogenetic clades (Fig. 1.I). Under this evolutionary frame, we discovered varieties of gene clusters, which may represent genes gained at two main evolutionary stages. The stage $\mathrm{I}$ is when $B$. anthracis and its sister species within the first phylogenetic clade diverged from bacillus species in the other two phylogenetic clades (Fig. 1.II). The stage II is when $B$. anthracis differentiated from $B$. cereus and B. thuringiensis, its closely related, non-anthrax species within the $B$. anthracis, B. cereus, and B. thuringiensis clade (Fig. 1.III \&1.IV). In following sections, we illustrated genes gained at each stage and highlighted those that might have potential impacts on the pathogenesis of $B$. anthracis.

Stage I is corresponding to a cluster of 717 genes that are present only in the genomes of the $B$. anthracis, $B$. cereus, and $B$. thuringiensis clade. Compared with pathogenic species in this clade, bacillus species in other two are nonpathogenic. B. licheniformis and B. subtilis belong to the Bacillus subtilis group and are commonly found soil bacteria. B. halodurans and B. clausii are widely distributed alkali-tolerant bacillus species. Analysis of the 717-gene cluster indicated that the divergence of the B. anthracis, $B$. cereus, and $B$. thuringiensis clade from other non-pathogenic clades appeared to be a critical point in the adaptive evolution of $B$. anthracis. Many genes gained in this stage have homologues of pathogenic importance. Among them, genes in Amino acid transport and metabolism [E] is the most dominant gene group gained at this stage (Fig. 2 ). It includes 10 genes that encode oligopeptide $A B C$ transporter, oligopeptide-binding proteins, a gene group that are required for bacterial growth at low temperature and involved in intracellular survival [26]. 


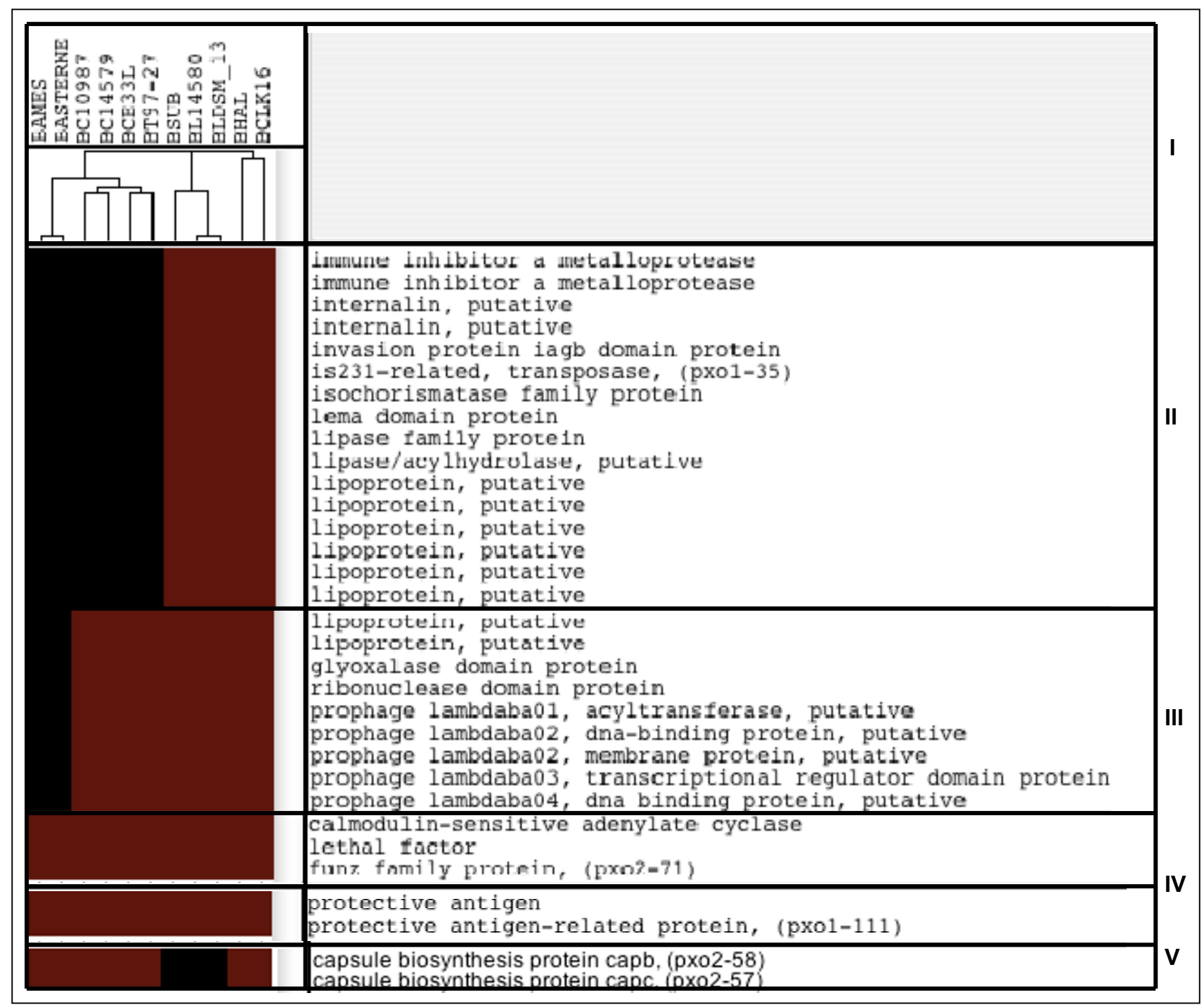

Figure I

The clustering analysis of genes gained by B. anthracis. The clustering analysis of $B$. anthracis-specific genes that were gained by $B$. anthracis. Genes included are those with obvious function annotations. Colour scheme: red stands for the absence of the genes and black for the presence. Note that genomes in the tree are labelled by their abbreviations: BAMES stands for $B$. anthracis Ames, BANSTR for B. anthracis Ames Ancestor 058I, BASTERNE for B. anthracis str. Sterne, BCI0987 for B. cereus ATCC 10987, BCI4579 for B. cereus ATCC I4579, BCE33L for B. cereus E33L, BT97-27 for B. thuringiensis konkukian str. 9727, BLI4580 for B. licheniformis ATCC I4580, BLDSM_I 3 for B. licheniformis DSM_I 3 ATCC I4580, BSUB for B. subtilis subsp. Subtilis str. 168 , BHAL for B. halodurans C-I 25 and BCLKI 6 for B. clausii KSM-KI 6

Cell wall/membrane/envelope biogenesis [M] is the second one. It covers, among others, genes for two internalin proteins, invasion protein iagB domain protein, and Slayer protein. The acquisitions of internalin proteins confers the pathogen ability to cross significant host barriers for entering, reside in, and multiply in phagocytic and non-phagocytic cells [27-30]. The Invasion protein iagB domain protein has homologues that are involved in invasion of HeLa cells by Salmonella enterica subsp. enter- ica ser. Typhi [31]. The S-layer protein, together with 13 other S-layer proteins that were classified into the unknown COG group, is one of the largest gene groups gained at this stage. The S-layer proteins are generally known to be self-assembled into a supramolecular structure enclosing the bacterial cells. The structure strategically positions to interact with the tissues and body fluids of the host and contributes to the outcome of a host-parasite interaction. In the fish-pathogenic bacterium Aerom- 


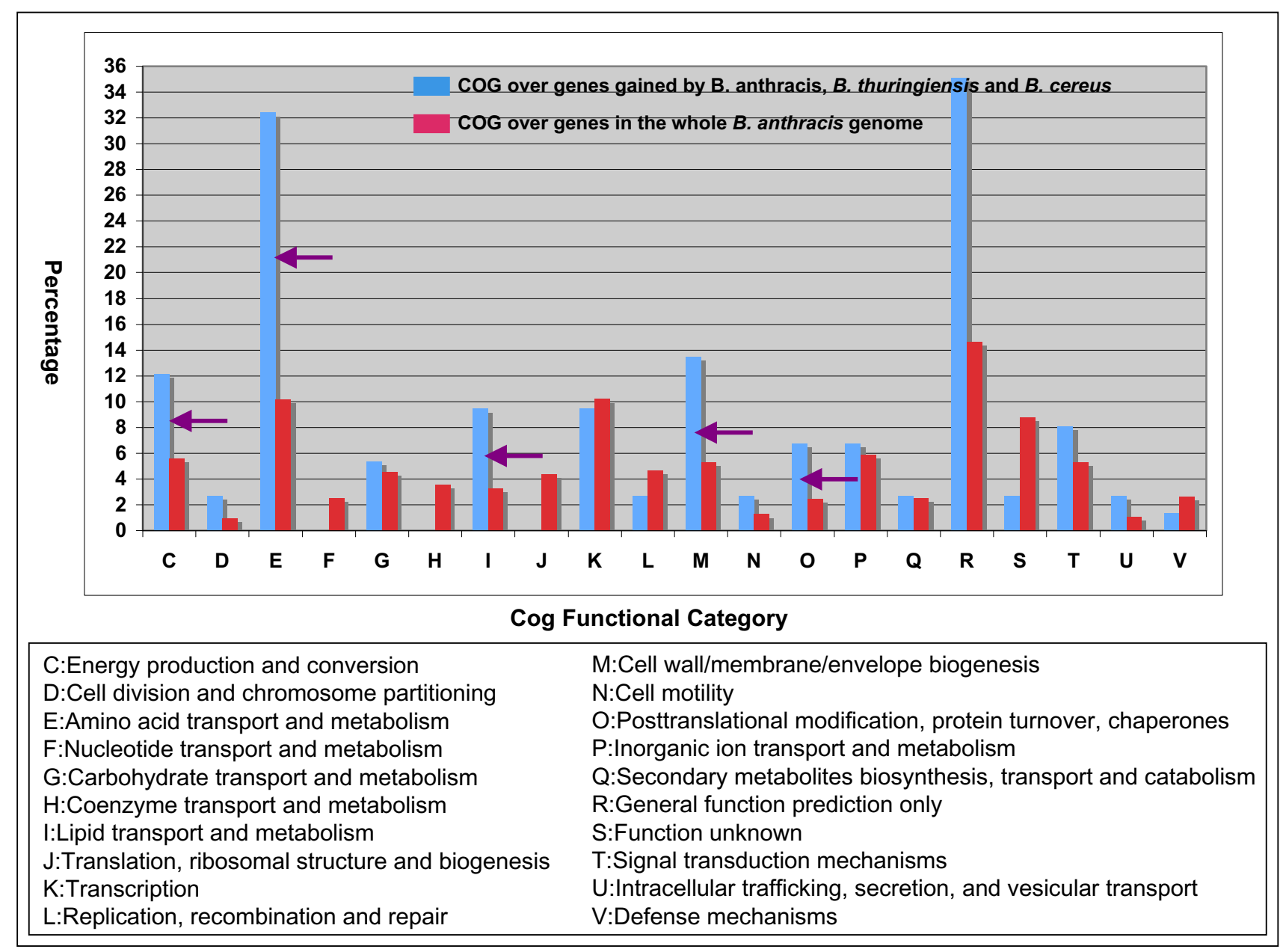

\section{Figure 2}

Comparative display of the COG occurrences of genes gained at stage I over those in the entire genome of $B$. anthracis Ames Ancestor $058 \mathrm{I}$. Comparative display of the COG occurrences of genes gained at stage I (blue bars) over those in the entire genome of $B$. anthracis Ames Ancestor 058I (red bars). The Y-coordinate represents the percentage of the gene occurrences in given COGs. Purple arrows represent those with the selective gene gains.

onas salmonicida, the array of the surface proteins, for example, dramatically enhances the virulence of the bacterium [32]. In the pathogenic strains of Aeromonas hydrophila and Aeromonas veronii biotype sobria, the surface-exposed and non-surface-exposed epitopes of the Slayer protein provide antigenic diversity [33].

The cluster furthermore includes homologues of genes known to encode the enhancin family protein [COG unsigned], two non-haemolytic enterotoxins [COG unsigned], an immune inhibitor A metalloprotease protein [S], two microbial collagenase proteins [R], up to 27 lipoprotein-coding genes [COG unassigned]. All the genes are related to pathogenesis of infectious agents if not directly to the bacterial pathogen. The enhancin, found in baculoviruses, is a metalloprotease that can boost viral infectivity by degrading the mucin layer surrounding insect guts [34]. The immune inhibitor A metalloprotease enhances virulence in insects through the cleavage of bacteriocidal lectins [35]. The homologues of non-haemolytic enterotoxins are known to be involved in B. cereus and B. thuringiensis pathogenesis [18]. The lipoprotein, the largest gene group gained at this stage, is a diverse group of proteins with covalent lipid modifications by fatty acids and other lipid moieties. The lipid-modified proteins often play diverse roles from surface adhesion to the translocation of virulence factors into the host cytoplasm. MxiM, a lipoprotein of the type III secretory pathway in Shigella flexneri, for example, is important for translocation of invasions [36], and MAA1 of Mycoplasma arthritidis, a surface-exposed lipoprotein, is required for adherence to joint tissues early in the infectious process 
[37]. From that, we were reasoning that gene gains at this stage would build crucial genetic foundations that allowed B. anthracis to evolve later as an anthrax pathogen that can enter, survive and replicate within the hash host cell systems.

Stage II includes two clusters with 83 (Fig. 1.III) and 93 genes (Fig. 1.IV) respectively. Since the clusters represent the difference between $B$. anthracis and its closely related, non-anthrax Bacillus species: $B$. cereus and B. thuringiensis, the acquisition of these genes by $B$. anthracis likely represents a recent event in the bacillus evolution. Between two clusters in this stage, genes in 83-gene cluster are present in all genomes of $B$. anthracis, thus specific to $B$. anthracis. The majority of them have no functional annotations except for those encoding two lipoproteins, one glyoxalase domain protein, one ribonuclease domain protein and several prophage lambdaba04 proteins. The genes in the 93-gene cluster are all plasmid genes (pX01 and pX02) and, among them are anthrax-factors such as lethal factor, protective antigen-related protein, protective antigen and calmodulin-seneitive adenylate cyclase. Although they are present only in B. anthracis Ames Ancestor 0581, we still considered them as B. anthracis-specific or anthrax-causing Bacillus pathogen-specific. The reason is that the pX01 and pX02 are considered an integrated part of the anthrax-causing Bacillus pathogen and $B$. anthracis Ames Ancestor 0581 is the only one that has the two plasmids and is pathogenic. While the above three clusters follow the evolution relationships in their gene occurrences, the cluster that contains the capsule biosynthesis protein capC and capB does not (Fig. 1.V). These two virulent factors exist only in $B$. Subtilis subsp. Subtilis str. 168, B. licheniformis DSM_13 ATCC 14580, B. licheniformis ATCC 14580 and B. anthracis Ames Ancestor 0581. While the capsule proteins are encoded by plasmid (pX02) in B. anthracis Ames Ancestor 0581, their homologues in the other three genomes are chromosomal, suggesting a usual mode of gene transmission: from chromosome to plasmids or vise verse.

\section{Analysis of gene losses}

We chose B. licheniformis ATCC 14580 as the reference genome to study the lineage-specific gene losses at stage I when $B$. anthracis and its sister species within the $B$. anthracis clade were diverged from Bacillus species in the other two phylogenetic clades. The analysis found a total of 103 genes that are absent in the genomes within the $B$. anthracis, B. cereus and B. thuringiensis clade but present in all the genomes from other two non-pathogenic clades. Among them, carbohydrate transport and metabolism $(\mathrm{G})$ is the most predominant functional group, including genes encoding two pectate lyases, polysaccharide lyase family 1 proteins, five glycoside hydrolase family proteins and two L-arabinose isomerase proteins. Cell motility (N) is the second, including multiple genes for flagellar components such as flagellar protein FliS, flagellar hook-basal body protein and CheD chemotaxis protein.

The study further selected B. cereus ATCC 10987 as the reference genome to study the lineage-specific gene losses at stage II when $B$. anthracis was differentiated from its two closest relatives. The genome was selected for its close kinship with B. anthracis and its characteristic plasmid [38]. The plasmid shares a basic skeleton with pX01, one of the virulent factor-associated plasmids in the anthrax-causing pathogen. The analysis found 184 genes lost from $B$. anthracis. Among them, 23 are present in all genomes of $B$. cereus and B. thuringiensis, but not in those of B. anthracis. Those include two two-gene groups of IS3-family transposase-coding genes, one regucalcin family proteincoding gene, and three lipoprotein-coding genes. The rest of the 184 genes vary in their presence in the genomes of $B$. cereus or $B$. thuringiensis but are consistently absent from those of $B$. anthracis. For example, genes that code three DNA recombinases, three spore coat proteins, and one transcriptional regulator, MarR family protein are present in the genomes of $B$. cereus only; genes that code two type I restriction-modification system, $M$ subunits and acid-soluble spore protein $\mathrm{P}$ are present in $B$. cereus ATCC 14579 and B. cereus ATCC 10987 only. The detection of the selective gene losses suggested possible roles in the Bacillus evolution and further experiments are necessary for their validations.

\section{Verification of the gene gains and losses}

With a set of nine additional bacillus genomes, we examined gene gains and losses at both stages. The results indicated that the inclusion of these genomes did not upset the occurrence patterns of the lineage-specific gene losses and gains except a 119-gene set that were gained at stage I (Fig. 3). These genes are absent in the genome of $B$. cereus subsp. cytotoxis NVH 391-98 but present all other bacillus genomes within the Bacillus cereus group. Among them are those that encode two internalins, cytolysin immunity Cyli domain protein, beta-lactamase II, betalactam antibiotic acylase family protein, trifolitoxin immunity domain protein, 11 putative lipoproteins, and nine acetyltransferases. The B. cereus strain, isolated in 1998 from an outbreak that caused fatal enteritis, is genetically remote from other $\mathrm{B}$. cereus group strains and highly pathogenic $[39,40]$. It will be interesting to know how the losses impact on this Bacillus strain and its cytotoxicity.

At the end of this section, we felt that we need to make some clarifications on our newly devised evolution-based scheme and some frequent terms used in the paper. First, we used Blast with a predetermined cutoff to determine gene losses and gains. The results indicated that this is a 


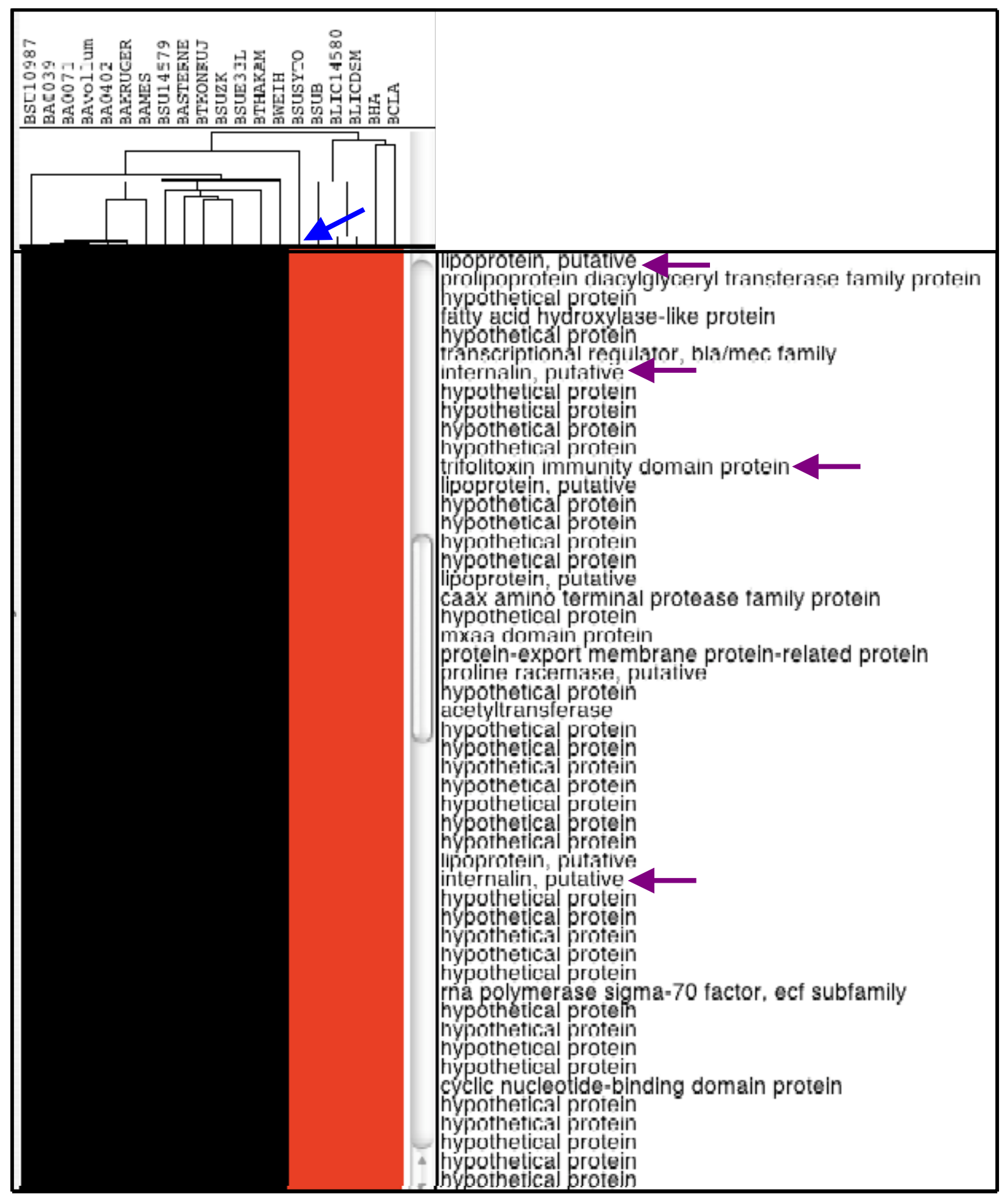

Figure 3

Display of genes that are absent in the genome of B. cereus subsp. cytotoxis NVH $391-98$ but present in all other bacillus genomes within the Bacillus cereus group. Displays of genes that are absent in the genome of $B$. cereus subsp. cytotoxis NVH 39I-98 (highlighted by Blue arrow) but present in all other bacillus genomes within the Bacillus cereus group. Purple arrows represent two internalin and other pathogenesis-related genes. The name abbreviations for newly added B. anthracis genomes are as followings: BA0039 for B. anthracis A0039, BA0402 for B. anthracis A0402, BAKROGER for B. anthracis str. Kruger B, BAVOLLUM for B. anthracis Vollum, and BA007I for B. anthracis A007I Western North America. 
simplified but valid approach as illustrated above where unique and pathogenically important genes were revealed from this analysis. The drawback is that the results can be compromised if homologous genes exist within the target genomes. One solution is to adjust the threshold. A minor problem is that sequence similarities between orthologs can vary from gene to gene. Alternatively, orthologs can be defined and used in the clustering analysis [41]. Second, we used "gained" to describe genes unique to $B$. anthracis but had no intension to distinguish whether they were actually "acquired" by B. anthracis or "pseudogenized" from its compared genomes.

\section{Conclusion}

The analysis presented here allowed us to portray a progressive evolutionary process during the lifestyle shift of $B$. anthracis from a free soil bacterium to an obligate pathogen. Selective gene losses and gains appeared to be one of the main driving forces underlying the adaptive evolution of $B$. anthracis. First, novel genes including those of pathogenic importance were lost/gained when $B$. anthracis, $B$. cereus and $B$. thuringiensis, the genomes within the Bacillus cereus group, were differentiated from $B$. licheniformis and other Bacillus genomes outside the Bacillus cereus group (stage I). Gene losses/gains further occurred in B. anthracis when this species diverged from its two closest relatives. Overall, our analysis provided new insights into how $B$. anthracis had evolved and bore a promise of finding drug and vaccine targets for this strategically important pathogen.

\section{Competing interests}

The author declares that they have no competing interests.

\section{Authors' contributions}

GXY is the sole contributor to this paper, covering all research processes from algorithm developments, data collections to data analysis.

\section{Acknowledgements}

This publication was made possible by NIH Grant \#P20 RR0 6454 from the INBRE Program of the National Center for Research Resources and by the Start-up fund from Boise State University

This article has been published as part of BMC Bioinformatics Volume 10 Supplement I, 2009: Proceedings of The Seventh Asia Pacific Bioinformatics Conference (APBC) 2009. The full contents of the supplement are available online at http://www.biomedcentral.com/ |47/ -2 |05//0?issue=SI

\section{References}

I. Beres SB, Richter EW, Nagiec MJ, Sumby P, Porcella SF, DeLeo FR, Musser JM: Molecular genetic anatomy of inter- and intraserotype variation in the human bacterial pathogen group $A$ Streptococcus. Proc Natl Acad Sci USA 2006, I 03( ( 8):7059-7064.

2. Rodriguez JL, Palmer GH, Knowles DP Jr, Brayton KA: Distinctly different msp2 pseudogene repertoires in Anaplasma marginale strains that are capable of superinfection. Gene 2005, 36I: I27-I32.
3. Sumby P, Whitney AR, Graviss EA, DeLeo FR, Musser JM: GenomeWide Analysis of Group A Streptococci Reveals a Mutation That Modulates Global Phenotype and Disease Specificity. PLoS Pathog 2006, 2(I):4I-49.

4. Thomson NR, Yeats C, Bell K, Holden MT, Bentley SD, Livingstone M, Cerdeno-Tarraga AM, Harris B, Doggett J, Ormond D, et al.: The Chlamydophila abortus genome sequence reveals an array of variable proteins that contribute to interspecies variation. Genome Res 2005, 15(5):629-640.

5. Andersson SG, Kurland CG: Reductive evolution of resident genomes. Trends Microbiol 1998, 6(7):263-268.

6. Parkhill J, Thomson N: Evolutionary strategies of human pathogens. Cold Spring Harb Symp Quant Biol 2003, 68: I5I-I58.

7. Wernegreen JJ: Genome evolution in bacterial endosymbionts of insects. Nat Rev Genet 2002, 3( I I):850-86I.

8. Gómez-Valero L, EP R, Latorre A, Silva FJ: Reconstructing the ancestor of Mycobacterium leprae: the dynamics of gene loss and genome reduction. Genome Res 2007, I7(8): I I78-I I 85.

9. Mira A, Ochman H, Moran NA: Deletional bias and the evolution of bacterial genomes. Trends Genet 200I, 17(1 0):589-596.

10. Maurelli AT: Black holes, antivirulence genes, and gene inactivation in the evolution of bacterial pathogens. FEMS Microbiol Lett 2007, 267(I): I-8.

II. Wilson BA, Salyers AA: Is the evolution of bacterial pathogens an out-of-body experience? Trends Microbiol 2003, I I (8):347-350.

12. Day WA, Maurelli AT: Evolution of Microbial Pathogens. ASM Press; 2006.

13. Marston CK, Gee JE, Popovic T, Hoffmaster AR: Molecular approaches to identify and differentiate Bacillus anthracis from phenotypically similar Bacillus species isolates. $B M C$ Microbiol 2006, 6():22-.

14. Sternbach G: The history of anthrax. J Emerg Med 2003, 24(4):463-467.

15. Guidi-Rontani C, Levy M, Ohayon H, Mock M: Fate of germinated Bacillus anthracis spores in primary murine macrophages. Mol Microbiol 200I, 42(4):93I-938.

16. Guidi-Rontani C, Weber-Levy M, Labruyere E, Mock M: Germination of Bacillus anthracis spores within alveolar macrophages. Mol Microbiol 1999, 31 (I):9-17.

17. Shafazand S, Doyle R, Ruoss S, Weinacker A, Raffin TA: Inhalational anthrax: epidemiology, diagnosis, and management. Chest 1999, I 16(5): 1369-1376.

18. Read TD, Peterson SN, Tourasse N, Baillie LW, Paulsen IT, Nelson KE, Tettelin H, Fouts DE, Eisen JA, Gill SR, et al.: The genome sequence of Bacillus anthracis Ames and comparison to closely related bacteria. Nature 2003, 423(6935):8I-86.

19. Rasko DA, Ravel J, Økstad OA, Helgason E, Cer RZ, Jiang L, Shores KA, Fouts DE, Tourasse NJ, Angiuoli SV, et al:: The genome sequence of Bacillus cereus ATCC 10987 reveals metabolic adaptations and a large plasmid related to Bacillus anthracis pXOI. Nucleic Acids Res 2004, I I (32):977-988.

20. Han CS, Xie G, Challacombe JF, Altherr MR, Bhotika SS, Brown N, Bruce D, Campbell CS, Campbell ML, Chen J, et al.: Pathogenomic sequence analysis of Bacillus cereus and Bacillus thuringiensis isolates closely related to Bacillus anthracis. I Bacteriol 2006, I88(9):3382-3390.

21. Genome Assembly/Annotation Projects] [ftp://ftp.ncbi.nih.gov/ genomes/Bacteria]

22. De Hoon MJL, Imoto S, Nolan J, Miyano S: Open source clustering software. Bioinformatics 2004, 20(9): 1453-1454.

23. Saldanha AJ: Java Treeview - extensible visualization of microarray data. Bioinformatics 2004, 20( 17 ):3246-3248.

24. Comprehensive Microbial Resource [ftp://ftp.jcvi.org/pub/data/ Microbial Genomes]

25. Xu D, Cote JC: Phylogenetic relationships between Bacillus species and related genera inferred from comparison of $3^{\prime}$ end I6S rDNA and 5' end I6S-23S ITS nucleotide sequences. Int J Syst Evol Microbiol 2003, 53(Pt 3):695-704.

26. Borezee E, Pellegrini E, Berche P: OppA of Listeria monocytogenes, an oligopeptide-binding protein required for bacterial growth at low temperature and involved in intracellular survival. Infect Immun 2000, 68( I 2):7069-7077.

27. Fedhila S, Daou N, Lereclus D, Nielsen-LeRoux C: Identification of Bacillus cereus internalin and other candidate virulence 
genes specifically induced during oral infection in insects. Mol Microbiol 2006, 62(2):339-355.

28. Olier M, Garmyn D, Rousseaux S, Lemaitre JP, Piveteau P, Guzzo J: Truncated internalin $A$ and asymptomatic Listeria monocytogenes carriage: in vivo investigation by allelic exchange. Infect Immun 2005, 73(I):644-648.

29. Olier M, Pierre F, Rousseaux S, Lemaitre JP, Rousset A, Piveteau P, Guzzo J: Expression of truncated Internalin $A$ is involved in impaired internalization of some Listeria monocytogenes isolates carried asymptomatically by humans. Infect Immun 2003, 7 I(3): I $217-1224$.

30. Werbrouck H, Grijspeerdt K, Botteldoorn N, Van Pamel E, Rijpens N, Van Damme J, Uyttendaele M, Herman L, Van Coillie E: Differential inIA and inIB expression and interaction with human intestinal and liver cells by Listeria monocytogenes strains of different origins. Appl Environ Microbiol 2006, 72(6):3862-387I.

31. Miras I, Hermant D, Arricau N, Popoff MY: Nucleotide sequence of iagA and iagB genes involved in invasion of HeLa cells by Salmonella enterica subsp. enterica ser. Typhi. Res Microbiol 1995, I 46(I): I7-20

32. Janda JM, Oshiro LS, Abbott SL, Duffey PS: Virulence markers of mesophilic aeromonads: association of the autoagglutination phenomenon with mouse pathogenicity and the presence of a peripheral cell-associated layer. Infect Immun 1987, 55( I 2):3070-3077.

33. Kostrzynska M, Dooley JS, Shimojo T, Sakata T, Trust TJ: Antigenic diversity of the S-layer proteins from pathogenic strains of Aeromonas hydrophila and Aeromonas veronii biotype sobria. J Bacteriol I992, I 74(I):40-47.

34. Lepore LS, Roelvink PR, Granados RR: Enhancin, the granulosis virus protein that facilitates nucleopolyhedrovirus (NPV) infections, is a metalloprotease. J Invertebr Pathol 1996, 68(2): $|3|-\mid 40$.

35. Cossart P: Molecular and cellular basis of the infection by Listeria monocytogenes: an overview. Int J Med Microbiol 2002, 29 I(6-7):40|-409.

36. Schuch R, Maurelli AT: The Mxi-Spa Type III Secretory Pathway of Shigella flexneri Requires an Outer Membrane Lipoprotein, MxiM, for Invasin Translocation. Infect Immun. 1999, 62(4):|982-199|.

37. Washburn LR, Miller EJ, Weaver KE: Molecular characterization of Mycoplasma arthritidis membrane lipoprotein MAAI. Infect Immun 2000, 68:437-442.

38. Rasko DA, Ravel J, Okstad OA, Helgason E, Cer RZ, Jiang L, Shores KA, Fouts DE, Tourasse NJ, Angiuoli SV, et al.: The genome sequence of Bacillus cereus ATCC 10987 reveals metabolic adaptations and a large plasmid related to Bacillus anthracis pXO I. Nucleic Acids Res 2004, 32(3):977-988.

39. Fagerlund A, Ween O, Lund T, Hardy SP, Granum PE: Genetic and functional analysis of the cytK family of genes in Bacillus cereus. Microbiology 2004, I 50(Pt 8):2689-2697.

40. Sorokin A, Candelon B, Guilloux K, Galleron N, Wackerow-Kouzova $N$, Ehrlich SD, Bourguet $D$, Sanchis V: Multiple-locus sequence typing analysis of Bacillus cereus and Bacillus thuringiensis reveals separate clustering and a distinct population structure of psychrotrophic strains. Appl Environ Microbiol 2006, 72(2): $1569-1578$

41. Overbeek R, Fonstein M, D'Souza M, Pusch GD, N M: The use of gene clusters to infer functional coupling. Proc Natl Acad Sci USA I999, 96(6):2896-290।.
Publish with Bio Med Central and every scientist can read your work free of charge

"BioMed Central will be the most significant development for disseminating the results of biomedical research in our lifetime. "

Sir Paul Nurse, Cancer Research UK

Your research papers will be:

- available free of charge to the entire biomedical community

- peer reviewed and published immediately upon acceptance

- cited in PubMed and archived on PubMed Central

- yours - you keep the copyright

Submit your manuscript here:

http://www.biomedcentral.com/info/publishing_adv.asp
BioMedcentral 